\title{
Encouraging top-down attention in visual search:A developmental perspective
}

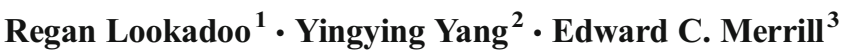

Published online: 17 July 2017

(C) The Psychonomic Society, Inc. 2017

\begin{abstract}
Four experiments are reported in which 60 younger children (7-8 years old), 60 older children (10-11 years old), and 60 young adults (18-25 years old) performed a conjunctive visual search task ( 15 per group in each experiment). The number of distractors of each feature type was unbalanced across displays to evaluate participants' ability to restrict search to the smaller subset of features. The use of top-down attention processes to restrict search was encouraged by providing external aids for identifying and maintaining attention on the smaller set. In Experiment 1, no external assistance was provided. In Experiment 2, precues and instructions were provided to focus attention on that subset. In Experiment 3, trials in which the smaller subset was represented by the same feature were presented in alternating blocks to eliminate the need to switch attention between features from trial to trial. In Experiment 4, consecutive blocks of the same subset features were presented in the first or second half of the experiment, providing additional consistency. All groups benefited from external support of top-down attention, although the pattern of
\end{abstract}

Portions of this research were conducted in partial fulfillment of the requirements of the $\mathrm{PhD}$ degree at the University of Alabama by the first author.

This research was partly supported by the Fundamental Research Funds for the Central Universities (Award No. 16wkpy26) and National Natural Science Foundation of China (Award No. 31600898) awarded to Y.Y.

Yingying Yang

yangyy35@mail.sysu.edu.cn

1 Georgetown College, Georgetown, KY, USA

2 Department of Psychology, Sun Yat-sen University, 135 Xingang West Road, Guangzhou 510275, China

3 The University of Alabama, Tuscaloosa, AL, USA improvement varied across experiments. The younger children benefited most from precues and instruction, using the subset search strategy when instructed. Furthermore, younger children benefited from blocking trials only when blocks of the same features did not alternate. Older participants benefited from the blocking of trials in both Experiments 3 and 4, but not from precues and instructions. Hence, our results revealed both malleability and limits of children's top-down control of attention.

Keywords Children · Visual search · Top-down attention . Subset search

The increasing ability to effectively control what aspects of the environment we attend to and how we attend to them is a critical feature of developmental change (Bjorklund \& Harnishfeger, 1990; Huang-Pollock, Maddox, \& Karalunas, 2011; Korkman, Kemp, \& Kirk, 2001; Lehman, Naglieri, \& Aquilino, 2010; Merrill \& Conners, 2013; Plude, Enns, \& Brodeur, 1994). For adults and children, attention can be drawn by exogenous features of the environment that are highly salient because of, for example, brightness or movement (Dannemiller, 1998; Theeuwes, 1991; Yantis \& Jonides, 1984). Attention can also be endogenously guided based on the goals, knowledge, and experience of the individual (Jonides \& Irwin, 1981; Posner, 1980; McCormick, 1997). Research has revealed little or no change in exogenously controlled attention over a wide age range (Brodeur, Trick, \& Enns, 1997; Enns, 1990, Rueda et al., 2004; Trick \& Enns, 1998). In contrast, researchers generally agree that endogenously controlled attention undergoes a great deal of developmental change that extends over a prolonged period of development (e.g., Enns \& Brodeur, 1989; Rueda, 2014; Rueda, Posner, \& Rothbart, 2005). 
One approach to studying the development of attention is to investigate age-related changes in visual search. In fact, the results of studies using visual search are highly consistent with the general pattern of attentional development. When attention can be directed on the basis of exogenous cues, as in single feature search when the search target differs from a set of homogeneous nontargets by a single and unique feature, individuals of all ages can find the target very efficiently (e.g., Merrill \& Conners, 2013; Trick \& Enns, 1998). Under these conditions, the target seems to "pop out" (Treisman \& Gelade, 1980), and response times do not vary as a function of number of items to be searched. In contrast, when endogenous control is required, large age differences are typically reported (Donnelly et al., 2007; Trick \& Enns, 1998). For example, a conjunctive search involves trying to locate a target that is formed by a conjunction of two features of the nontargets, such as looking for a blue circle in a field of yellow circles and blue squares. In this case, attention is purposefully and systematically deployed to search through the individual items to find the target. Younger children find this much more difficult than older children and adults. Hence, the younger children take longer per item to search an array for the target (e.g., Merrill \& Lookadoo, 2004; Trick \& Enns, 1998).

Developmental research assessing performance in a conjunctive search task has also revealed more refined age-related differences in the efficient allocation of attention. More specifically, it is clear that adults can guide attention during search to specific features of an array on the basis of feature distinctiveness and current goals (see Egeth, Virzi, \& Garbart, 1984; Zohary \& Hochstein, 1989). In one demonstration of this ability, Egeth et al. (1984) had participants search for a red $O$ embedded in a display that included distracters of black $O$ s and red $N \mathrm{~s}$. The researchers held one subset of distracters at a constant and smaller number and gradually increased the number of distracters in the other subset. Participants were also instructed to restrict their search to the smaller subset. Search slopes in this condition were much less steep than in the condition where the number of distracters in both subsets increased at the same time. They therefore concluded that their participants were able to limit search to the constant and smaller size subset, because search times did not increase as a function of the total number of distracters in the display. Zohary and Hochstein (1989) subsequently demonstrated that search is fastest when the difference in the number of items in the two subsets is large, especially in comparison to when there is an equal number of each type of distracter, a phenomenon called the distracter-ratio effect.

Merrill and Lookadoo (2004) investigated this effect developmentally. They compared the performance of 7-year-old and 10-year-old children to adults in a conjunctive search task where the target was a black circle embedded in distracters of gray circles and black squares. Across two experiments, they varied the ratio of black and gray distracters in the search displays. Based on search rates, Merrill and Lookadoo concluded that all participants benefited from searching a display that included a small subset of items with a salient feature (i.e., two black square distracters and the black circle target in displays of 15 or 25 total items). In fact, the 10 -year-old and adult participants exhibited no interference at all from the added number of gray circle distracters and the 7-year-old participants exhibited only a small portion of the interference typically found for adding additional items to the search array. However, as the number of black items in the smaller subset increased to, for example, five items, the performance of the children began to suffer relative to that of the adults. The adults could still restrict search to the more salient black distracters, as evidenced by slope values that increased as the number of black distracters increased but not as the number of gray distracters increased. However, the children could no longer do so, as their search rates increased with the increase in the number of gray distracters in the search display. Apparently, younger and older children could limit search to a small subset of the overall display only if that subset was sufficiently small (i.e., three out of 15) so as to be distinct from other items in the display. This suggested that preattentive processes may have worked to help guide attention to those items (see Wolfe, 1994; Wolfe, Cave, \& Frankel, 1989). However, when the black and gray items were more balanced in number (e.g., five of 15 items) and likely required effort to selectively attend to one type of distracter relative to the other, only the adults were able to effectively restrict their search to that subset.

One of the questions not answered by previous research is whether attentional control problems alone account for the performance differences observed between the children and adults in studies such as Merrill and Lookadoo (2004). It may also be that search strategy differences contributed to producing the developmental differences. For example, younger children may fail to recognize the value of restricting attention to a subset of distracters or have difficulty implementing a strategy that is available to them under some stimulus conditions. The current research was conducted to address this question. The plan was to evaluate whether or not it is possible to improve the search efficiency of children through a set of stimulus changes and explicit instructions. Therefore, rather than simply identifying activities in which children perform poorly, the results of the four experiments reported here provide data relevant to understanding children's transition from being relatively weak to relatively strong at coordinating attention in support of visual search activities. In addition, these studies may provide useful data that can help to identify difficulties with general top-down attentional control experienced by individuals of all ages outside of visual search (e.g., Gaspelin, Margett-Jordan, \& Ruthruff, 2015; Matusz et al., 2015; Remington, Cartwright-Finch, \& Lavie, 2014). In particular, they address the utility of different forms of external assistance in improving children's and adult's control of visual attention. 


\section{Current study}

In the current study, younger children (7-8 years), older children (10-11 years), and young adults took part in four experiments. In each experiment, participants were required to determine whether or not a target defined in terms of the conjunction of color (blue) and shape (circle) was present in a display of blue squares and green circles. Most of the displays contained an unbalanced number of each type of distracter. In some displays, the smaller subset consisted of blue squares, and in some they were green circles. The four experiments differed in the degree of external assistance that was provided to encourage efficient search of the smaller subset.

Experiment 1 was essentially a replication of Merrill and Lookadoo (2004), in which no external assistance was provided to encourage participants to limit search to the smaller subset. Hence, we expected that the basic results would be similar to that study, with young adults being generally good and younger children being relatively bad at restricting search to the smaller subset.

In Experiment 2, we encouraged the use of a smaller subset search strategy through the presentation of cues. Presenting informative cues about the feature of a target has been shown to improve conjunction search efficiency in adults under conditions in which the specific target is uncertain (e.g., Anderson, Heinke, \& Humphreys, 2012, 2013; Zhuang \& Papathomas, 2011). It is reasonable to expect that cues that identify the relevant target feature of smaller subset, combined with explicit instructions to use these cues, may help improve search efficiency in children. Cues would likely serve multiple functions for the children. First, although identifying and selectively attending to the smaller subset may be relatively automatic in adults (Kim \& Cave, 1999; Shen, Reingold, \& Pomplun, 2003; Sobel, $\&$ Cave, 2002), it may be more effortful for children to recognize that the ratio of distracter types is different. The cue would thus alert them to this information and allow them to use it during search. Second, children's less efficient performance in selective subset search may be because they are unaware that attending to the smaller subset is a more efficient search strategy than attending to all the stimuli, especially when the difference is not extreme (e.g., two vs. 20). Receiving explicit instructions about the usefulness of attending the small subset may help children direct their attention to the small subset and increase their search efficiency to a level comparable to that of adults. Hence, we used precues to designate which target feature was associated with the smaller subset (a BLUE filled rectangular bar or a black CIRCLE), as well as instructing participants to search the smaller set designated by the cue. If the children perform as well as adults under these conditions, then we would attribute their difficulties to strategy selection processes rather than being able to focus attention on the smaller subset. If their performance is still somewhat less efficient than that of the adults, then other mechanisms of attention would be implicated.
In Experiments 3 and 4, we encouraged the development of an appropriate attentional set by providing displays of a single type in blocks of trials where the critical target feature of the smaller subsets did not change (e.g., color or shape), instead of intermixing them. It may be that younger children both recognize and are able to restrict attention to the smaller subset on some trials but are unable to maintain that focus over trials because the identity of the feature defining the smaller subsets changed from trial to trial. Blocking displays for presentation may allow young children to develop an attentional set that is easier to maintain across trials. Zohary and Hochstein (1989) suggested that adult participants were able to switch the selectively attended subset from trial to trial based on the feature of the small subset to exhibit maximum search efficiency. Later studies (e.g., Shen, Reingold, \& Pomplun, 2000, 2003; Williams \& Reingold, 2001) also demonstrated that adult participants' saccades were biased toward the smaller subset regardless of its shared feature with the target (cf. Poisson \& Wilkinson, 1992, for an alternative account of subset search efficiency). It is likely that the attentional shifting process, which may require relatively little effort for adults, is relatively more demanding for children as demonstrated in other cognitive domains, such as executive function (e.g., Lee, Bull, \& Ho, 2013; Lehto, Juujärvi, Kooistra, \& Pulkkinen, 2003). Hence, if blocking similar displays can reduce the cognitive load associated with switching subsets from trial to trial in children, then children's search efficiency should also increase relative to adults. In Experiment 3, trials in which the smaller subset was represented by the same feature were presented in alternating blocks to eliminate the need to switch attention between features from trial to trial. In Experiment 4, consecutive blocks of the same subset features were presented in the first or second half of the experiment providing additional consistency.

\section{Experiment 1}

\section{Method}

Participants There were 15 younger children ( $M=7.8$ years, $S D=.7$ years; eight females and seven males), 15 older children $(M=10.3$ years, $S D=.8$ years; seven females and eight males), and 15 adults (18-25 years old; nine females and six males). The children were recruited from local public schools. The college participants were undergraduate students in introductory psychology classes who received course credit for taking part. All recruitment and testing procedures followed the Institutional Review Board guidelines of the university.

Materials. Stimulus displays consisted of one target (blue circle) and two types of distracters: blue squares and green circles. Each individual stimulus subtended approximately $0.75^{\circ}$ of 
visual angle in height and width at a distance of $30 \mathrm{~cm}$ from the monitor. Stimuli were displayed in a $10^{\circ} \times 10^{\circ}$ visual angle white square presented in the center of the monitor. Displays could include eight, 16, or 32 total items. Throughout the experiments, half of the displays included a target and half did not.

The total number of items in the smaller subset had to be half (as an upper bound) or less than half of the total number. The actual ratios of smaller subset to total items was 4 of 8,4 of 16,4 of 32,8 of 16,8 of 32 , and 16 of 32 across the six ratio conditions. The smaller subset was always defined in terms of the individual features of the target. Hence, the smaller subset could be "blue items" relative to a larger set of "green items" or "circle items" relative to a larger set of "square items." In the target-absent displays, the smaller set consisted of all distracters - for example, four blue squares or four green circles. In the target-present condition, one item in the smaller set was replaced by the target resulting in, for example, three blue squares and one blue circle for four blue items and three green circles and one blue circle for four circle items. Figure 1 provides an example of each subset condition for the 4 of 16 ratio displays.

There were 384 trials in total. An equal number (i.e., 64 trials) of displays were presented in each distracter ratio condition. Within each distracter ratio condition, there were equal number of target-present and target-absent displays (i.e., 32 trials each). Finally, for each set of these 32 trials, 16 included a smaller subset of circle items and 16 included a smaller subset of blue items.

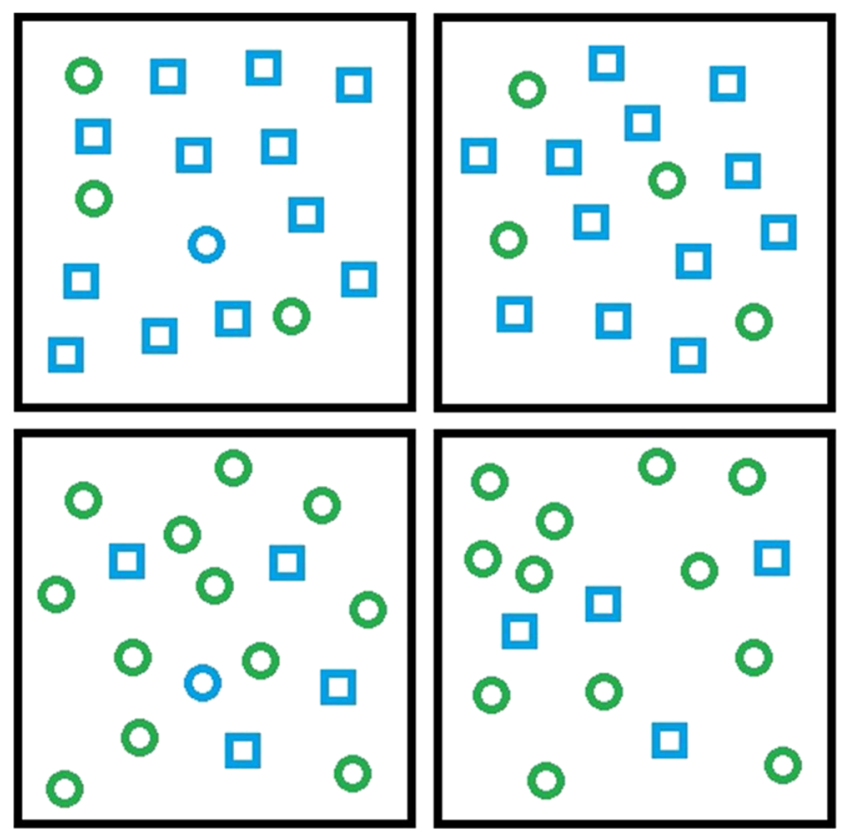

Fig. 1 Examples of stimulus displays in the 4 of 16 ration conditions. Top displays represent displays in which CIRCLE was the smaller subset, and bottom displays represent displays in which BLUE was the smaller subset. On the left were target-present displays and on the right were target-absent displays. (Color figure online)
Design. The primary variables in the experiment were group (younger children, older children, and adults), subset size (four and eight), and response type (target present and target absent). Subset size and response type were manipulated within participants. The primary dependent variable was response time to determining whether or not a target was in each display. Response times (to the nearest ms) and errors were recorded automatically.

Procedure. The experiment was presented using Superlab program software. Participants were told to search each display for a blue circle and press the target-present key of the response box if they found the target and the target-absent key if they did not. Each display remained visible until a response was made. Both speed and accuracy were emphasized. Participants received up to 30 practice trials before the experimental trials began. All displays were presented in random order.

\section{Results}

All analyses were conducted on raw scores and repeated on scores following log transformation to address potential issues of distribution nonnormality. There was only one difference in results across all experiments, as noted below in Experiment 3 . For brevity, we only report results for the analysis of raw scores.

Data from the 16 of 32 ratio condition were not included in the analyses. This condition was included to balance the design in the eyes of the participants. However, because the primary analyses were based on intercept and slope values calculated for the smaller subsets and the 16 item subset occurred in only one ratio condition, intercept and slope values using this condition could not be calculated for the primary analyses. Additionally, this ratio condition was also associated with more errors (about 10\%), especially in children's groups, and therefore considered less informative concerning RT differences. Errors in the experimental conditions were relatively rare $(2.5 \%$ for the younger children, $3.0 \%$ for the older children, and $1.6 \%$ for the adults). Error rates were analyzed using Group $\times$ Target (present vs. absent) ANOVA. The analysis indicated a main effect of target, $F(1,42)=85.28, p<.001$, $\eta_{\mathrm{p}}{ }^{2}=.670$, with more errors in the target-present condition (3.7\%) than in the target-absent condition (1.5\%). Neither the main effect of group nor the interaction were significant. For the primary analyses, we computed slope and intercepts using a multiple regression analysis predicting response times for the total search set size (eight, 16, or 32) while controlling for the size of the smaller subset (four or eight). The primary analyses were based on the resulting coefficient for the total set size (slope values) and constant (intercept values).

The analysis of slope values assessed whether the number of items in the larger subset influenced search times. Separate 
analyses were conducted on the target-present and target-absent trials because performance on target-absent trials tend to be more susceptible to the influence on extraneous variables (e.g., response bias, decision making criteria) that can vary both within and across groups adding nonsystematic variability to the basic results (see, e.g., Merrill \& Lookadoo, 2004). More specifically, participants attempting to restrict search to the small subset would likely find a target in the smaller subset and end their search for target-present trials. Thus, slope values would be at or near zero ms because participants use the same strategy regardless of the overall number of distracters. However, not finding a target may lead to broadening the search to the rest of the display to ensure the correct decision is made, leading to larger slope values as a function of this strategic change in target-absent trials. Hence, including both targetpresent and target-absent slopes in the same analysis may be misleading. Intercept values reflect time required to perform operations that happened once during the task (e.g., decision making, response). Although they were not particularly relevant to assess top-down control of attention, the focus of this research, they were also analyzed here as a measure of overall RT differences. Data are presented in Table 1.

Slope values Slope values were analyzed separately for target-present and target-absent trials using a one-way ANOVA, with group as the independent variable and slope values as the dependent variable. The analysis of targetpresent trials yielded a significant effect of group, $F(2,42)=$ 7.95, $p=.01, \eta_{\mathrm{p}}{ }^{2}=.275$. The younger children exhibited significantly greater slope values than did either the older children or the adults. The two older groups did not differ from each other. Hence, the younger children were significantly less able to restrict search to the smaller subset in the target-present condition of Experiment 1. The analysis of target-absent slopes was not significant, $F(2,42)=.12, \eta_{\mathrm{p}}{ }^{2}=.005$, with all groups exhibiting similar slope values. Apparently, the ability to restrict attention was limited to finding a target. If a target was not found, either the participants searched the

Table 1 Slope and intercept values for Experiment 1 as a function of group and response type (SDs in parentheses)

\begin{tabular}{llllll}
\hline \multirow{2}{*}{ Group } & \multicolumn{2}{l}{ Slope } & & & Intercept \\
\cline { 2 - 3 } \cline { 5 - 6 } & Present & Absent & & Present & Absent \\
\hline Younger & 12.5 & 19.3 & & 981 & 1111 \\
& $(8.1)$ & $(12.6)$ & & $(144)$ & $(301)$ \\
Older & 6.0 & 18.6 & & 808 & 793 \\
& $(4.0)$ & $(11.9)$ & & $(120)$ & $(215)$ \\
Adult & 5.1 & 20.5 & & 614 & 521 \\
& $(3.4)$ & $(8.8)$ & & $(94)$ & $(169)$ \\
\hline
\end{tabular}

smaller subset several more times or searched the entire display to be certain they did not miss the target. Both would result in steeper slopes in the target-absent condition. These results are generally consistent with those of Merrill and Lookadoo (2004), who also found a significant age difference for target-present but not for target-absent trials. Despite the relatively smaller slope values obtained in the target-present condition, the slope value for each group was significantly greater than zero, $t(14)=5.99,5.90$, and 5.88 for the younger children, older children, and adults, respectively; all $p \mathrm{~s}<.001$.

Intercept values. Intercept values were also analyzed separately for target-present and target-absent trials using a oneway ANOVA, with group as the independent variable and intercept values as the dependent variable. The effect of group was significant for target-present trials, $F(2,42)=34.30, p<$ $.01, \eta_{\mathrm{p}}{ }^{2}=.620$, with all groups being significantly different from each other (all $p$ s $<.05$ using Tukey's HSD). The effect of group was also significant for target-absent trials, $F(2,42)=$ 23.61, $p<.01, \eta_{\mathrm{p}}{ }^{2}=.529$. Again, all groups were significantly different from each other (all $p \mathrm{~s}<.05$ using Tukey's HSD). Hence, children were slower to respond overall, and adults were fastest.

\section{Experiment 2}

Experiment 2 was designed to encourage the use of top-down attentional processes by identifying the small subset for the participant prior to the appearance of the display. If inefficient subset search in Experiment 1, particularly associated with the performance of the younger children, was due to participants' failure to identify the smaller set prior to initiating their search, then this manipulation would facilitate performance in Experiment 2.

\section{Method}

Participants. We recruited 15 new participants in each of the three age groups. The mean age of the younger children was 7.7 years $(S D=.6$; seven females and eight males) and the older children was 10.5 years $(S D=.5$; nine females and six males). The adults ranged in age from 18 to 25 years (nine females and six males).

Materials and procedures. The stimuli were identical to Experiment 1. The procedure was changed such that Experiment 2 included precues and verbal instructions on each trial. We did not attempt to separate these two pieces of information because it seemed that providing precues without telling them what to do with the information would not make any sense to the participants. For each trial, a cue appeared first for $1,000 \mathrm{~ms}$ prior to the presentation of the search 
display. There were two types of cues: a color cue and a shape cue. The color cue was a filled, blue bar. Although the color cue also had a shape, its shape was very different from those in the search displays. Hence, it was not expected to confuse participants into thinking it was a shape cue. The shape cue was a black outline circle. Because black was not a color present in the search displays, only shape information from this cue was relevant for the participants. The color cue was only paired with displays where the small or equal subset was of blue items and the shape cue was only paired with the displays where the small or equal subset was of circle items. When the two subsets had equal set sizes, each cue appeared half of the time. Because the cue always predicted the feature of the smaller or equal subset, these cues would increase search efficiency if used.

A total of 384 displays were again presented in random order. In addition to the general instructions, participants were explicitly told to focus their search for the target to the subset of the items in the display to which they were directed by the instruction cue. The experimenter made sure that the child participants understood the use of the precues before starting the experiment.

\section{Results}

Again, errors in the experimental conditions were relatively rare (2.0\% for the younger children, $2.2 \%$ for the older children, and $1.9 \%$ for the adults). The Group $\times$ Target analysis indicated a main effect of target presence, $F(1,42)=18.71, p$ $<.001, \eta_{\mathrm{p}}^{2}=.3 .08$, with more errors in the target-present condition (2.4\%) than in the target-absent condition (1.6\%). Neither the main effect of group nor the interaction were significant. The primary analyses were identical to Experiment 1. Data used in the analyses are presented in Table 2.

Slope values. As in Experiment 1, slope values were analyzed separately for target-present and target-absent trials using a one-way ANOVA. For the target-present data, slope values were not significantly different as a function of age group, $F(2,42)=1.53, p>.10, \eta_{\mathrm{p}}^{2}=.070$. For the target-absent data, similar to Experiment 1, the slope values again were not significantly different across age groups, $F(2,42)=.35, \eta_{\mathrm{p}}{ }^{2}=$ .016. In fact, the magnitude of the target-absent slopes were essentially identical in Experiment 2 and Experiment 1. Hence, younger children benefited from precues and instructions only in the target-present trials, but the manipulation did not impact any other group or condition. However, as in Experiment 1, all target-present slope values were significantly different from zero, $t(14)=5.82,7.09$, and 8.64 for the younger children, older children, and adults, respectively; all $p \mathrm{~s}<.001$. Thus, again, none of the groups were able to fully restrict search to the smaller subset.
Table 2 Slope and intercept values for Experiment 2 as a function of group and response type ( $S D \mathrm{~s}$ in parentheses)

\begin{tabular}{llllll}
\hline \multirow{2}{*}{ Group } & \multicolumn{2}{l}{ Slope } & & & Intercept \\
\cline { 2 - 3 } \cline { 6 - 6 } & Present & Absent & & Present & Absent \\
\hline \multirow{2}{*}{ Younger } & 8.6 & 20.6 & & 984 & 1156 \\
& $(5.0)$ & $(16.1)$ & & $(281)$ & $(620)$ \\
Older & 6.9 & 17.2 & & 801 & 844 \\
& $(3.3)$ & $(10.4)$ & & $(214)$ & $(199)$ \\
Adult & 6.2 & 20.8 & & 574 & 516 \\
& $(2.8)$ & $(11.0)$ & $(258)$ & $(170)$ \\
\hline
\end{tabular}

Intercept values. Similar to Experiment 1, analysis of intercept values revealed a significant effect of group for targetpresent trials, $F(2,42)=12.96, p<.01, \eta_{\mathrm{p}}{ }^{2}=.376$, with all groups being significantly different from each other (all $p$ s $<$ .05 using Tukey's HSD). There was also a significant difference for target-absent trials, $F(2,42)=21.25, p<.01, \eta_{\mathrm{p}}{ }^{2}=$ .497. Again, all groups were significantly different from each other (all $p$ s <.05 using Tukey's HSD). Hence, again, children were slower to respond overall and adults were fastest.

\section{Experiment 3}

The manipulations of Experiment 2 reduced the difference between ages. However, none of the groups were able to fully restrict search to the smaller subset, as indexed by the fact that slope values for all groups were still significantly greater than zero. In Experiment 3, we removed the need for participants to switch attention between features from one trial to the next by presenting the same small subset feature within blocks of trials in an attempt to further facilitate small subset search for all groups.

\section{Method}

Participants. Fifteen new participants in each of three age groups were recruited. Mean ages were 7.7 years for the younger children $(S D=.4$; nine females and six males) and 10.8 years for the older children $(S D=.6$; eight females and seven males). The age range of the adults was 18 to 25 years (nine females and six males).

Materials and procedures The stimuli were identical to Experiment 2. The procedure was changed such that Experiment 3 involved precues and verbal instructions, plus we blocked displays in which smaller subset shared the same feature. The 384 total trials were divided into four color blocks 
and four shape blocks, with 48 trials in each block. Within each block, there were four target-present and four targetabsent trials for each of six ratio conditions (i.e., 4 of $8 ; 4$ of $16 ; 4$ of $32 ; 8$ of $16 ; 8$ of $32 ; 16$ of 32 ). In the color blocks, the cues were always color cues, and the smaller subset in the displays was always composed of blue items. In the shape blocks, the cues were always shape cues, and the smaller subset in the displays was always composed of circle items. The displays within each block were randomized. The color and the shape blocks alternated during presentation. Whether each participant received color or shape blocks first was counterbalanced.

\section{Results}

Again, errors in the experimental conditions were relatively rare $(2.1 \%$ for the younger children, $3.1 \%$ for the older children, and $1.9 \%$ for the adults). None of the effects in the Group $\times$ Target analysis of error rates was significant. The primary analysis was identical to the previous experiments. Data used in the analyses are presented in Table 3.

Slope values. The analysis of target-present slopes indicated a significant effect of group, $F(2,42)=5.17, p<.01, \eta_{\mathrm{p}}{ }^{2}=.198$. The slope values of the younger children were significantly greater than those of the older children and the adults, who did not differ from each other. Interestingly, the pattern of group differences was more similar to Experiment 1 than to Experiment 2. Apparently, the blocking of trials improved the performance of the two older groups and not the younger group, which resulted in a significant difference in performance between groups on the target-present trials. For the first time, the analysis of target-absent trials also revealed a significant difference in slope values across groups, $F(2,42)=6.41$, $p<.01, \eta_{\mathrm{p}}{ }^{2}=.234$. Tukey's HSD revealed that all three groups were significantly different from each other (all $p s<.05$ ), with the younger children exhibiting the steepest slopes, the older children falling in the middle, and the adults exhibiting the

Table 3 Slope and intercept values for Experiment 3 as a function of group and response type (SDs in parentheses)

\begin{tabular}{llllll}
\hline \multirow{2}{*}{ Group } & \multicolumn{2}{l}{ Slope } & & & Intercept \\
\cline { 2 - 3 } \cline { 6 - 6 } & Present & Absent & & Present & Absent \\
\hline Younger & 7.2 & 24.9 & & 910 & 940 \\
& $(6.4)$ & $(25.1)$ & & $(229)$ & $(448)$ \\
Older & 3.3 & 12.2 & & 805 & 805 \\
& $(3.2)$ & $(13.5)$ & & $(186)$ & $(190)$ \\
Adult & 2.1 & 3.4 & & 620 & 672 \\
& $(3.0)$ & $(3.2)$ & & $(91)$ & $(139)$ \\
\hline
\end{tabular}

shallowest slope. Hence, the blocking manipulation appears to have altered the manner in which older children and adults searched displays without a target. Both groups appear to restrict search more efficiently in the target-absent trials of Experiment 3 than in the previous experiments, with the adult participants actually searching the displays without targets as efficiently as the displays with targets. Thus, older children and adults benefited from blocking in both target-present and target-absent trials whereas younger children did not benefit in either condition. However, despite the clear improvement in search efficiency observed in Experiment 3, the performance of all of the groups in the target-present trials was still significantly greater than zero, $t(14)=4.32, p<.001$, for the younger children; $t(14)=3.99, p<.001$ for the older children; and $t(14)=2.76, p=.015$ for the adults.

Intercept values. The analysis of intercepts for target-present trials indicated a significant effect of group, $F(2,42)=9.80, p$ $<.01, \mathrm{v}=.3 .18$. In this case, the younger children exhibited a significantly larger intercept value than the two older groups (both $p$ s $<.05$ using Tukey's HSD, but the two older groups did not differ significantly from each other. In the analysis of target-absent trials, there also was a significant effect of group, $F(2,42)=3.16, p=.05, \eta_{\mathrm{p}}{ }^{2}=.134$. However, this effect was not significant following the log transformation and is not considered further.

\section{Experiment 4}

In Experiment 3, the blocking of displays appears to have improved the performance of the adults and older children, but not the younger children. Both older groups were able to better restrict search to the smaller subset when they could establish an attentional set over trials. It may be that the fact that we alternated the feature that identified the smaller set across blocks limited the ability of the younger participants to develop the appropriate attentional set. Importantly, the younger children performed as well in Experiment 3 as they did in Experiment 2, indicating they were not confused by the additional manipulation. Hence, in Experiment 4, we modified the basic procedures in two additional ways to further encourage top-down guidance in the younger children. First, we presented all trials in which the subset was based on one feature (e.g., color) before presenting all trials based on the second feature (e.g., shape). This manipulation completely eliminated the need to switch attention set between several blocks and should therefore maximally encourage the development of an appropriate attentional set. Second, we provided feedback when an incorrect response was made (which by default provides feedback for correct responses, as well). Although incorrect responses were relatively rare in the study, feedback was expected to increase participants' confidence in 
their performance and elicit faster overall response times. In addition to these substantive changes, we decided to increase the number of trials to provide an opportunity to evaluate whether any remaining group differences were susceptible to practice effects. Taken together, these manipulations should provide the younger children with the greatest likelihood of exhibiting top-down control of attention to focus on the smaller subset in the displays.

\section{Method}

Participants Fifteen new participants for each age group took part in the study. The mean age of the younger children was 7.6 years ( $S D=.4$; seven females and eight males). The mean age of the older children was 10.8 years $(S D=.9 ; 11$ females and four males). Adults ranged in age from 18 to 20 years (eight females and seven males).

Materials and procedures The stimuli were identical to Experiment 1. The procedure was changed such that Experiment 4 was divided into two segments. Each consisted of four blocks of 72 trials per block. Within each segment, the small subset was the same in each block (i.e., either blue items or circle items). Prior to the start of the blue cue session, participants received instructions to search only among blue items for the target. They were also told that there would be fewer blue items in the displays, and hence it would be more efficient to search only among blue items. Similarly, prior to the start of the circle cue segment, participants were told to search only among the circle items for the target. The order of segments was counterbalanced between participants within each age group. After each block of 72 trials, there was a short break, and participants were reminded of the smaller subset. There were six target-present and six target-absent trials for each ratio condition in each block (i.e., 4 of $8 ; 4$ of $16 ; 4$ of 32 ; 8 of $16 ; 8$ of $32 ; 16$ of 32). There were four color blocks and four shape blocks for a total of 576 trials. When participants made a wrong response, the word wrong would appear after the response. Feedback was not provided when participants made correct responses.

\section{Results}

As was the case in the previous experiments, error rates in the experimental conditions were relatively low $(4.0 \%$ for the younger children, $2.9 \%$ for the older children, and $2.5 \%$ for the adults). Errors were analyzed using a Group $\times$ Epoch $(1,2$, 3 , and 4) $\times$ Target Presence ANOVA. The analysis indicated a significant effect of target presence, $F(1,42)=18.71, p<.001$, $\eta_{\mathrm{p}}{ }^{2}=.3 .08$, with more errors in the target-absent (3.5\%) than in the target-present condition (2.7\%). No other effects were significant. For the primary analyses, to increase statistical power we combined two blocks into one epoch resulting in
12 trials for each unique condition in each epoch. Search slopes and search intercepts are presented in Tables 4, 5, 6, and 7.

Slope values. Slope values for the target-present trials were analyzed using a 3 (group: adults, older children, younger children $) \times 4$ (epoch) mixed ANOVA. Greenhouse-Geisser method was used wherever appropriate. None of the effects were significant. Averaging across the four epochs, the slope values were $.4 \mathrm{~ms}$ per item for adults, $.5 \mathrm{~ms}$ for older children, and $.3 \mathrm{~ms}$ for younger children. Similarly, for the target-absent trials, there were no significant effects (although the main effect of epoch approached significance, $p=.064$ ). Averaging across four the epochs, the slope values were $5.1 \mathrm{~ms}$ per item for adults, $7.2 \mathrm{~ms}$ for older children, and $9.3 \mathrm{~ms}$ for younger children. Again, we evaluated whether slope values in the target-present conditions were significantly greater than zero for each group. In Experiment 4, none of the target-present slope values was significantly greater than zero (all $p \mathrm{~s}>.10$ ), indicating they were generally successful at restricting search to the smaller subset.

Intercept values. For the target-present condition, the main effect of group was significant, $F(2,42)=12.28, p<.001, \eta_{\mathrm{p}}{ }^{2}$ $=.369$, with younger children $(1,002 \mathrm{~ms})$ having significantly larger intercepts than older children $(716 \mathrm{~ms})$ and adults $(696$ $\mathrm{ms}$ ), who did not differ from each other. The main effect of epoch was also significant, $F(2.02,84.74)=7.92, p<.001$, $\eta_{\mathrm{p}}{ }^{2}=.159$, with significantly smaller intercepts observed for later presented epochs. The interaction was not significant, $F(4.04,84.74)=.61, \eta_{\mathrm{p}}{ }^{2}=.021$. For the target-absent condition, the main effect of group was significant, $F(2,42)=9.74$, $p<.001, \eta_{\mathrm{p}}^{2}=.318$, with younger children $(1107 \mathrm{~ms})$ having significantly larger intercepts than older children $(767 \mathrm{~ms})$ and adults $(716 \mathrm{~ms})$, who did not differ from each other $(p s<.05)$. The main effect of epoch was significant, $F(2.05,84.00)=$ $3.94, p=.02, \eta_{\mathrm{p}}{ }^{2}=.06$, with significantly smaller intercepts in Epochs 3 and 4 than for Epochs 1 and 2. The interaction was not significant, $F(3.65,76.54)=0.78, \eta_{\mathrm{p}}^{2}=.036$.

Table 4 Intercept values for Experiment 4 in the target-absent conditions as a function of group and epoch (SDs in parentheses)

\begin{tabular}{lllll}
\hline & Epoch 1 & Epoch 2 & Epoch 3 & Epoch 4 \\
\hline Younger children & 1187 & 1214 & 874 & 1155 \\
& $(570)$ & $(454)$ & $(403)$ & $(496)$ \\
Older children & 906 & 778 & 690 & 695 \\
& $(591)$ & $(273)$ & $(210)$ & $(233)$ \\
Adults & 846 & 729 & 662 & 628 \\
& $(570)$ & $(262)$ & $(196)$ & $(137)$ \\
\hline
\end{tabular}


Table 5 Slope values for Experiment 4 in the target-absent conditions as a function of group and epoch ( $S D \mathrm{~s}$ in parentheses)

\begin{tabular}{lllll}
\hline & Epoch 1 & Epoch 2 & Epoch 3 & Epoch 4 \\
\hline Younger children & 14.3 & 9.1 & 10.6 & 3.3 \\
& $(30.1)$ & $(20.9)$ & $(20.1)$ & $(9.1)$ \\
Older children & 14.7 & 5.3 & 5.5 & 3.3 \\
\multirow{4}{*}{ Adults } & $(15.5)$ & $(11.7)$ & $(7.2)$ & $(4.9)$ \\
& 8.7 & 4.3 & 5.6 & 2.1 \\
& $(14.8)$ & $(7.8)$ & $(7.3)$ & $(4.1)$ \\
\hline
\end{tabular}

\section{Cross-experiment analyses}

We conducted a Group $\times$ Experiment ANOVA on the targetpresent and target-absent slope values to evaluate the impact of the various manipulations used across experiments. We used the last epoch of Experiment 4 (rather than the average across epochs) as our index of performance for that experiment. The analysis of target-present trials indicated a significant main effect of group, $F(2,165)=9.22, p<.001, \eta_{\mathrm{p}}{ }^{2}=$ .101 , a significant main effect of Experiment, $F(3,165)=$ $31.28, p<.001, \eta_{\mathrm{p}}{ }^{2}=.363$, and a significant interaction, $F(6,165)=2.52, p=.02, \eta_{\mathrm{p}}{ }^{2}=.085$. The analysis of targetabsent trials also indicated a significant main effect of experiment, $F(3,165)=18.53, p<.001, \eta_{\mathrm{p}}{ }^{2}=.252$, and a significant interaction of Group $\times$ Experiment, $F(6,165)=3.15, p=$ $.006, \eta_{\mathrm{p}}{ }^{2}=.103$. The main effect of group was not significant, $F(2,165)=2.68, p=.071, \eta_{\mathrm{p}}^{2}=.032$. To compare the impact of our manipulations on each group, we subsequently compared changes in subset slope values across experiments for each group separately. Because we were specifically interested in improvements associated with each subsequent manipulation, following a significant overall result for target-present or target-absent trials for the group, we compared slope values in each experiment with the next using individual $t$ tests.

For the younger children, the analysis of target-present trials revealed a significant effect of experiment, $F(3,56)=$ $11.26, p<.001, \eta_{\mathrm{p}}{ }^{2}=.376$. A series of $t$ tests revealed that slopes values were significantly smaller in Experiment 2 than

Table 6 Intercept values for Experiment 4 in the target-present conditions as a function of group and epoch ( $S D \mathrm{~s}$ in parentheses)

\begin{tabular}{lllll}
\hline & Epoch 1 & Epoch 2 & Epoch 3 & Epoch 4 \\
\hline Younger children & 1165 & 1031 & 903 & 906 \\
& $(374)$ & $(265)$ & $(433)$ & $(336)$ \\
Older children & 805 & 700 & 706 & 652 \\
& $(305)$ & $(178)$ & $(202)$ & $(206)$ \\
Adults & 810 & 710 & 650 & 614 \\
& $(265)$ & $(150)$ & $(104)$ & $(113)$ \\
\hline
\end{tabular}

in Experiment 1, $t(28)=1.84, p=.03$, one-tailed, not different between Experiments 2 and 3, and significantly smaller in Experiment 4 than in Experiment 3, $t(28)=3.78, p=.001$, one-tailed. The analysis of target-absent trials also revealed a significant effect of experiment, $F(3,56)=4.47, p=.005, \eta_{\mathrm{p}}{ }^{2}$ $=.203$. The $t$ tests indicated that the only significant reduction in slope values occurred between Experiments 3 and 4, $t(28)=$ $3.14, p=.004$, one-tailed. For the older children, the analysis of target-present slopes revealed a significant effect of experiment, $F(3,56)=14.59, p<.001, \eta_{\mathrm{p}}{ }^{2}=.439$. No difference was observed between slopes of Experiments 1 and 2 . However, there was a significant reduction in slopes between Experiments 2 and 3, $t(28)=3.13, p=.004$, one-tailed, and between Experiments 3 and 4, $t(28)=3.48, p=.002$, onetailed. The analysis of target-absent trials was also significant, $F(3,56)=6.35, p<.001, \eta_{\mathrm{p}}{ }^{2}=.254$. Similar to the younger children, the older children only exhibited a reduction in slope values for absent trials between Experiments 3 and 4, $t(28)=$ $2.40, p=.012$, one-tailed. For the adults, the analyses also reveal an effect of Experiment for both the target-present trials, $F(3,56)=14.62, p<.001, \eta_{\mathrm{p}}{ }^{2}=.440$, and the target-absent trials, $F(3,56)=26.64, p<.001, \eta_{\mathrm{p}}{ }^{2}=.605$. For the targetpresent conditions, Experiments 1 and 2 were not different. However, a significant reduction in slope values was observed between Experiments 2 and 3, $t(28)=3.86, p<.001$, onetailed, and between Experiments 3 and 4, $t(28)=2.30, p=$ .015 , one-tailed. For the target-absent conditions, Experiments 1 and 2 were not different; Experiments 2 and 3 were significantly different, $t(28)=15.89, p<.001$, one-tailed; but Experiments 3 and 4 were not different. Hence, our manipulations did improve performance across experiments for each group, but the individual manipulations impacted each age group somewhat differently.

\section{Discussion}

In this study, we examined developmental differences in the deployment of attention during visual search. In particular, we were interested in participants' abilities to restrict attention to

Table 7 Slope values for Experiment 4 in target-present conditions as a function of group and epoch ( $S D$ s in parentheses)

\begin{tabular}{lllll}
\hline & Epoch 1 & Epoch 2 & Epoch 3 & Epoch 4 \\
\hline Younger children & -1.9 & 2.3 & 1.1 & -.5 \\
& $(11.3)$ & $(11.3)$ & $(9.0)$ & $(4.6)$ \\
Older children & 1.5 & 1.9 & -.8 & -.4 \\
& $(5.0)$ & $(4.7)$ & $(2.4)$ & $(2.6)$ \\
Adults & -.9 & 1.5 & 1.2 & -.2 \\
& $(3.7)$ & $(3.8)$ & $(2.2)$ & $(2.6)$ \\
\hline
\end{tabular}


the smaller of two subsets that contained the target and how selecting the appropriate subset and maintaining attentional focus may have accounted for the developmental differences in this process. Several differences were observed in the analysis of slope values. In Experiment 1where no external aids were provided, the younger children exhibited steeper slopes in the target-present condition than did the older children and adults. When aids to selecting the appropriate subset were provided in the form of precues and instructions in Experiment 2, the performance of the younger children was more similar to the older children and adults. In Experiment 3, trials were also blocked to eliminate the need to switch between features to identify the smaller set from trial to trial. Experiment 3 thus was designed to help maintain attentional focus on the small subset. Interestingly, the adults and older children were the ones who benefited the most from this manipulation. Older children were better able to restrict search in the target-present conditions and adults in both the targetpresent and target-absent conditions. In Experiment 4, when displays of the same small subset feature were presented in a single, longer segment, all three age groups were able to effectively direct attention to the smaller subset. This was true for the youngest children, even in the target-absent condition. With respect to intercept differences, our manipulations that were designed to impact search rate (slopes) had very little effect. Significantly smaller intercepts in adults relative to the youngest children were found in all four experiments and in both target-present and target-absent conditions.

Experiment 1 essentially replicated the results of Merrill and Lookadoo (2004) concerning children's ability to restrict attention to a smaller subset during visual search. More specifically, the younger children exhibited steeper slopes in the targetpresent condition than did the older children and adults. We reasoned that the inefficiency of children's search could reflect difficulties in selecting the appropriate subset, difficulties in maintaining attentional focus on the appropriate subset, or some combination of both. Both accounts are consistent with Merrill and Lookadoo (2004) and also with Merrill and Conners (2013), who reported that younger children had difficulty inhibiting irrelevant feature information during visual search. The presence of a feature that did not define the target (e.g., color) impacted the performance of younger children more than older children and adults in a feature search procedure. In addition, research by Woods et al. (2013) supports the possibility that younger children had more difficulty maintaining focus and organizing their search through the display. They found that 6- to 7-year-old children were less systematic in their search of displays (e.g., not using a left to right search pattern) and exhibiting a greater likelihood of revisiting previously searched locations. Experiments 2 and 3 considered these alternatives.

One difference between the results of Experiment 1 and those of Merrill and Lookadoo (2004) was that the older children were much more similar to the adults in the current study. It seems likely that this difference can be attributed to differences in the materials. Merrill and Lookadoo used black and gray circles and squares, whereas the current study used green and blue stimuli. One consequence of this choice is that child participants may have found it easier to contrast the stimuli on the basis of the color dimension. Hence, they were better able to focus search using top-down mechanisms when color identified the smaller subset set in Experiment 1 relative to using gray and black for that purpose in Merrill and Lookadoo (2004). This difference between experiments also highlights the interplay between bottom-up and top-down factors in visual search. Top-down mechanisms are only effective when bottom-up processes provide enough information to use them (Wolfe, 1994).

Experiment 2 included precues with instructions to help participants select and restrict search on the basis of either shape or color (depending on which subset was smaller). This manipulation increased the search efficiency of younger participants when there was a target in the display. This is important, especially considering that search slopes are typically very difficult to improve, especially in children (Vales, \& Smith, 2015). Essentially, the inclusion of precues and instructions provided top-down information of the identity of the small subset and helped direct attention to it prior to the appearance of the display. Hence, the search efficiency of the younger participants improved in Experiment 2 relative to Experiment 1 and was no longer significantly different from the other two older groups, indicating a greater ability to restrict search when provided external assistance to do so. The simplest explanation for this improvement is that young children have more difficulty identifying and initiating a search plan that is maximally efficient on their own than do older children and adults. The more inefficient search of the younger children in Experiment 1 could have been the result of at least two strategic choices. First, they may have consistently focused on the more salient dimension (e.g., color), regardless of which subset was smaller. This would be consistent with the basic principles of guided search (Wolfe, 2014). Second, they may have chosen a strategy for the current trial based on the previous trial. This later possibility may reflect a form of intertrial priming (e.g., Maljkovic \& Nakayama, 1994) where the younger participants base their current search strategy on the dimension that was most efficient for the previous trial. In contrast, older children and adults are more likely to base their current strategy on an evaluation of the current display.

It is interesting that the precues and instructions had no impact on participants' performance on the target-absent trials. Apparently, the possibility of missing a target encourages caution. Hence, to be certain that they did not miss the target, participants either searched the smaller subset additional times or searched the remaining items in the set. Traditionally, it was 
assumed that participants would likely search the entire display to conclude that there was no target present (e.g., Treisman \& Gormican, 1988; Treisman \& Souther, 1985), although data to the contrary have been observed (e.g., Humphreys, Quinlan, \& Riddoch, 1989). It was thought that providing cues and instructions might provide the impetus for developing a more efficient search strategy in the target-absent trials as well as the target-present trials. However, it appears that was not the case in Experiment 2. The possibility that the target may have been missed was sufficient to cause participants to revert to a less efficient but more certain strategy of searching the entire display. Further, this strategy choice did not vary as a function of participant age.

In Experiment 3, we added another level of certainty to the search process. Participants were not only made aware of which subset would be smaller, but they were given the opportunity to develop a processing set to search for a particular feature defined subset over trials. While it did result in relatively more efficient search overall, it did not effectively change the pattern of performance across age groups in the target-present trials. The adults and older children were able to restrict search to the smaller subsets more easily in Experiment 3. However, the younger children were not able to make use of the blocking procedure to effectively restrict attention any more than they did with precues and instructions in Experiment 2. Perhaps, despite the blocking of trials, the younger participants initiated their strategy anew each time they were shown the precue rather than developing an attentional set that could be used across trials. It may also be the case that the older participants benefited from positive effects of intertrial priming or repetition effects (e.g., Lamy \& Kristjansson, 2013; Maljkovic \& Nakayama, 1994) to a greater degree than did the younger children as a result of the blocking procedure. Unfortunately, the present data cannot address that possibility and will require further investigation. Regardless of the mechanism responsible, this is an important limitation on the ability of young children to allocate attention. Hence, while the results of Experiment 2 indicate that even the youngest participants have a basic ability to restrict attention to a subset of the display based on one of the two target features, Experiment 3 indicates that there are some important constraints on this ability.

In Experiment 4, we provided more consistent practice with the subset features by providing all trials associated with one target feature for four blocks of trials. In addition, we provided feedback when participants' responses were incorrect. These manipulations were sufficient to encourage even the youngest participants to restrict search to the smaller subset. In fact, all slope values in the targetpresent condition were near zero when the smaller subset was held constant. Because the zero slope values were obtained in the first epoch, it seems extended practice was not required to produce this change. Participants also did not exhibit a cost when the feature of the smaller subset changed between Epochs 2 and 3. Therefore, the consistent presentation was sufficient to allow the participants to develop and maintain the appropriate attentional set over time. Switching between subset features in Experiment 3, even with periods of consistent training prior to the switch, disrupted the ability to maintain that attentional focus in the younger children. In addition, the increased confidence in responding associated with the limited feedback provided greater trust in their rapid responses and therefore yielded flatter slopes with faster overall response times. We do not know if either manipulation alone would have been sufficient, only that together they were highly effective.

We also found that blocking the trials based on the target feature of the smaller subset encouraged the participants to end their search through the target-absent displays without necessarily searching the entire display. The adult participants actually searched the displays without targets as efficiently as they did the displays with targets in both Experiments 3 and 4. The older children exhibited somewhat smaller slopes in Experiment 3 and slopes that approached zero in the targetabsent condition of Experiment 4. The younger children exhibited the ability to restrict search in the target-absent conditions of Experiment 4. Hence, it appears that there are manipulations that can impact attention and performance criteria when targets are not present in the display as well. Still, it is important to note that influencing performance of targetabsent trials proved to be much more difficult than influencing performance of target-present trials. We suspect that confidence in knowing that the target was not missed is substantially more difficult to achieve than confidence knowing that a target was found. Further, reaching criterion for accepting that a target was not present changed with age. More specifically, the results suggested that the two older groups, but not the younger group, may have reached the necessary criterion with less blocked experience in Experiment 3. However, even the younger children reached the necessary criterion with enough blocked experience in Experiment 4.

Taken together, the results of these four experiments reveal some important strengths and weaknesses in the abilities of children to engage top-down guided attention. Importantly, even the 7- to 8-year-old participants could focus attention sufficiently to restrict search to the smaller subset when conditions were appropriate. Nevertheless, our study, together with several previous studies (Couperus, 2011; Couperus, Hunt, Nelson, \& Thomas, 2011; Yang \& Merrill, 2014, 2015) indicate that young children still exhibit numerous inefficiencies when they deploy attention in support of visual search. Further, the transition to exhibiting adult-like efficiency happens rapidly, with 10to 11-year-old children performing remarkably similarly to college students with much less experimenter-provided support. The approaches used to facilitate search efficiency in these 
studies, providing cues, instructions, and blocking very likely engaged children's top-down processing in support of identifying and selecting the smaller subset during visual search. It has been argued that the selection of the smaller subset often involves a bottom up process driven by feature saliency because it occurs even when the subset changes from trial to trial and can be amplified by increasing the contrast between features (Koch \& Ullman, 1985; Shen et al., 2003; Sobel \& Cave, 2002; Wolfe, 1994). However, top-down processes associated with knowledge and intention of the participants can also be used to effectively direct attention. In addition, improving top-down processing can assist attentional guidance in conjunction search (Wolfe, 1994; Wolfe et al., 1989). Several researchers have shown that providing instructions to college students about the feature of the small subset can improve their search reaction times (Shen, Elahipana, \& Reingold, 2007; see also Bacon \& Egeth, 1997; Egeth et al., 1984; Kaptein, Theeuwes, \& Van der Heijen, 1995; Sobel \& Cave, 2002). When cued by color, adults were not only able to restrict their search to the color elements of the display but were also able to switch from one subset to another from trial to trial as the cue switched from one color to another (Kaptein, et al., 1995).

Several mechanisms of learning and performance may help to account for the age-related differences we have observed. For example, we suspect that changes resulting from perceptual learning may play an important role. Except for the most basic features, children need to learn to distinguish between dimensions of features. In particular, Thompson and Massaro (1989) report that there is a growing ability to differentiate small differences among objects with age. In the case of the current study, being able to differentiate between two colors and two shapes with some general level of efficiency may be prerequisite for flexibly allocating attentional resources to search the smaller subset without aid. The younger children required assistance to do this, indicating a relative weakness in this aspect of their performance. Another important difference between younger children and adults is the degree to which younger children can maintain an appropriate attentional set in the face of changing conditions. Sustained, selective attention is one of several important executive functions that improve with age (Fisher \& Kloos, 2016). Given the reemergence of group differences in Experiment 3 when the small subset dimension remained the same across several trials, it appears that the younger children needed more assistance than older children and adults to maintain attention to a known smaller subset as well. Hence, we expect that the development of executive functions play an important role as well.

What we have observed with our manipulations also closely aligns with several features of general theories of development. In fact, our results are highly consistent with the concepts of production and utilization deficiencies in strategy use as described, for example, by Flavell (1970) and Bjorklund (Bjorklund, Miller, Coyle, \& Slawinski, 1997). Production deficiencies reflect a failure to spontaneously produce a strategy that would be useful. It seems that younger children were not able to spontaneously produce a subset search strategy in Experiment 1. However, when we provided the strategy and gave explicit instructions to focus on the smaller subset in Experiment 2, they were able to use it to improve their overall performance. A production deficiency was not evident for the older children and adults. It appears they spontaneously produced and used the strategy because instructions to do so did not change their search performance.

All three groups exhibited varying degrees of a utilization deficiency (Bjorklund et al., 1997). A utilization deficiency reflects a failure to fully benefit from a strategy even though it was produced. Clearly, all three groups required external support to make best use of the small subset search strategy. What changes with development is the amount of support necessary to engage and maintain the strategy when appropriate. With increasing age, there is an increased ability to generalize the strategy to situations where the conditions provide less support. Hence, the blocking of trials, especially in Experiment 4, provided additional benefits by reducing resource requirements (e.g., eliminating the need to switch attention between trials) and allowing the development of an attentional set (e.g., reducing memory requirements). In addition, providing feedback likely increased confidence in using the strategy. These manipulations facilitated the utilization of the subset search strategy for all participants, and for the older participants the manipulations also allowed the strategy to more readily generalize to target-absent trials.

\section{Conclusion}

In summary, our results indicate that it is possible to impact the visual search efficiency of young children in a way that allows them to perform as efficiently as young adults under a limited set of conditions. Provided with external assistance that encourages the use of top-down processes to control attention, young children were able to restrict attention to a small subset of the search display identified by a single feature of the target. However, they did not do so spontaneously. In addition, the older children and adults exhibited improvements in search efficiency in the most advantageous conditions such that they still outperformed the younger children. Hence, it appears that age-related differences may reflect more than strategic differences. There may be some attentional capacity differences that contribute to the developmental differences we observed in the top-down control of visual attention. Nevertheless, our results do suggest that children's attention during visual search can be modified by task conditions and may suggest a potential means for facilitating attentional processing in children with visual attention difficulties. 


\section{References}

Anderson, G. M., Heinke, D., \& Humphreys, G. W. (2012). Bottom-up guidance to grouped items in conjunction search: Evidence for color grouping. Vision Research, 52(1), 88-96. doi:10.1016/j.visres.2011. 11.011

Anderson, G. M., Heinke, D., \& Humphreys, G. W. (2013). Top-down guidance of eye movements in conjunction search. Vision Research, 7936-7946. doi:10.1016/j.visres.2012.12.008.

Bacon, W. F., \& Egeth, H. E. (1997). Goal-directed guidance of attention: Evidence from conjunctive visual search. Journal of Experimental Psychology: Human Perception and Performance, 23, 948-961.

Bjorklund, D. F., \& Harnishfeger, K. K. (1990). The resources construct in cognitive development: Diverse sources of evidence and a theory of inefficient inhibition. Developmental Review, 10(1), 48-71. doi: 10.1016/0273-2297(90)90004-N

Bjorklund, D. F., Miller, P. H., Coyle, T. R., \& Slawinski, J. L. (1997). Instructing children to use memory strategies: Evidence of utilization deficiencies in memory training studies. Developmental Review, 17(4), 411-441. doi:10.1006/drev.1997.0440

Brodeur, D., Trick, L., \& Enns, J. T. (1997). Selective attention over lifespan. In J. Burack \& J. Enns (Eds.), Attention, development, and psychopathology (pp. 74-93). New York: Guilford Press.

Couperus, J. W. (2011). Perceptual load influences selective attention across development. Developmental Psychology, 47, 1431-1439. doi:10.1037/a0024027

Couperus, J. W., Hunt, R. H., Nelson, C. A., \& Thomas, K. M. (2011). Visual search and contextual cueing: Differential effects in 10-yearold children and adults. Attention, Perception, \& Psychophysics, 73, 334-348. doi:10.3758/s13414-010-0021-6

Dannemiller, J. L. (1998). A competition model of exogenous orienting in 3.5-month-old infants. Journal of Experimental Child Psychology, 68, 169-201. doi:10.1006/jecp. 1997.2426

Donnelly, N., Cave, K., Greenway, R., Hadwin, J. A., Stevenson, J., \& Sonuga-Barke, E. (2007). Visual search in children and adults: Topdown and bottom-up mechanisms. The Quarterly Journal of Experimental Psychology, 60(1), 120-136. doi:10.1080/ 17470210600625362

Egeth, H. E., Virzi, R. A., \& Garbart, H. (1984). Searching for conjunctively-defined targets. Journal of Experimental Psychology: Human Perception and Performance, 10, 32-39.

Enns, J. T. (1990). Relations between components of visual attention. In J. T. Enns \& J. T. Enns (Eds.), The development of attention: Research and theory (pp. 139-158). Oxford, UK: Amsterdam, The Netherlands: North-Holland. doi:10.1016/S0166-4115(08) 60454-9

Enns, J. T., \& Brodeur, D. A. (1989). A developmental study of covert orienting to peripheral visual cues. Journal of Experimental Child Psychology, 48, 171-189.

Fisher, A., \& Kloos, H. (2016). Development of selective and sustained attention: The role of executive functions. In J. A. Griffin, P. McCardle, \& L. S. Freund (Eds.), Executive function in pre-school aged children: Integrating measurement, neurodevelopment, and translational research (pp. 215-237). Washington, DC: APA. doi: 10.1037/14797-010

Flavell, J. (1970). Developmental studies of mediated memory. In H. W. Reese \& L. P. Lipsitt (Eds.), Advances in child development and behavior (Vol. 5, pp. 181-211). New York, NY: Academic Press.

Gaspelin, N., Margett-Jordan, T., \& Ruthruff, E. (2015). Susceptible to distraction: Children lack top-down control over spatial attention capture. Psychonomic Bulletin \& Review, 22(2), 461-468. doi:10. 3758/s13423-014-0708-0

Huang-Pollock, C. L., Maddox, W. T., \& Karalunas, S. L. (2011). Development of implicit and explicit category learning. Journal of
Experimental Child Psychology, 109(3), 321-335. doi:10.1016/j. jecp.2011.02.002

Humphreys, G. W., Quinlan, P. T., \& Riddoch, M. J. (1989). Grouping processes in visual search: Effects with single- and combinedfeature targets. Journal of Experimental Psychology: General, 118(3), 258-279. doi:10.1037/0096-3445.118.3.258

Jonides, J., \& Irwin, D. E. (1981). Capturing attention. Cognition, 10(1/3), 145-150. doi:10.1016/0010-0277(81)90038-X

Kaptein, N. A., Theeuwes, J., \& Van der Heijden, A. H. C. (1995). Search for a conjunctively defined target can be selectively limited to a color-defined subset of elements. Journal of Experimental Psychology: Human Perception and Performance, 21, 1053-1069.

Kim, M., \& Cave, K. R. (1999). Top-down and bottom-up attentional control: On the nature of interference from a salient distractor. Perception \& Psychophysics, 61(6), 1009-1023. doi:10.3758/ BF03207609

Koch, C., \& Ullman, S. (1985). Shifts in selective visual attention: Towards the underlying neural circuitry. Human Neurobilogy, 4, 219-227.

Korkman, M., Kemp, S. L., \& Kirk, U. (2001). Developmental assessment of neuropsychological function with the aid of the NEPSY. In A. S. Kaufman, N. L. Kaufman, A. S. Kaufman, \& N. L. Kaufman (Eds.), Specific learning disabilities and difficulties in children and adolescents: Psychological assessment and evaluation (pp. $347-$ 386). New York: Cambridge University Press. doi:10.1017/ CBO9780511526794

Lamy, D. F., \& Kristjánsson, Á. (2013). Is goal-directed attentional guidance just intertrial priming? A review. Journal of Vision, 13(3), 14 14. doi: $10.1167 / 13.3 .14$

Lee, K., Bull, R., \& Ho, R. H. (2013). Developmental changes in executive functioning. Child Development, 84(6), 1933-1953. doi:10. 1111/cdev.12096

Lehman, E. B., Naglieri, J. A., \& Aquilino, S. A. (2010). A national study on the development of visual attention using the Cognitive Assessment System. Journal of Attention Disorders, 14(1), 15-24. doi: $10.1177 / 1087054709332473$

Lehto, J. E., Juujärvi, P., Kooistra, L., \& Pulkkinen, L. (2003). Dimensions of executive functioning: Evidence from children. British Journal of Developmental Psychology, 21(1), 59-80. doi: 10.1348/026151003321164627

Maljkovic, V., \& Nakayama, K. (1994). Priming of pop-out: I. Role of features. Memory \& Cognition, 22(6), 657-672. doi:10.3758/ BF03209251

Matusz, P. J., Broadbent, H., Ferrari, J., Forrest, B., Merkley, R., \& Scerif, G. (2015). Multi-modal distraction: Insights from children's limited attention. Cognition, 136, 156-165. doi:10.1016/j.cognition.2014. 11.031

McCormick, P. A. (1997). Orienting attention without awareness. Journal of Experimental Psychology: Human Perception and Performance, 23, 168-180.

Merrill, E. C., \& Conners, F. A. (2013). Age-related interference from irrelevant distracters in visual feature search among heterogeneous distracters. Journal of Experimental Child Psychology, 115(4), 640 654.

Merrill, E. C., \& Lookadoo, R. (2004). Selective search for conjunctively defined targets by children and young adults. Journal of Experimental Child Psychology, 89(1), 72-90.

Plude, D. J., Enns, J. T., \& Brodeur, D. (1994). The development of selective attention: A life-span overview. Acta Psychologica, 86(2/3), 227-272. doi:10.1016/0001-6918(94)90004-3

Poisson, M. E., \& Wilkinson, F. (1992). Distractor ratio and grouping processes in visual conjunction search. Perception, 21, 21-38.

Posner, M. I. (1980). Orienting of attention. Quarterly Journal of Experimental Psychology, 32, 3-25.

Remington, A., Cartwright-Finch, U., \& Lavie, N. (2014). I can see clearly now: The effects of age and perceptual load on inattentional 
blindness. Frontiers in Human Neuroscience, 8. doi:10.3389/ fnhum.2014.00229

Rueda, M. R. (2014). Development of attention. In K. N. Ochsner, S. M. Kosslyn, K. N. Ochsner, \& S. M. Kosslyn (Eds.), The Oxford handbook of cognitive neuroscience (Core topics, Vol. 1, pp. 296-318). New York, NY: Oxford University Press.

Rueda, M. R., Fan, J., McCandliss, B. D., Halparin, J. D., Gruber, D. B., Lercari, L. P., \& Posner, M. I. (2004). Development of attentional networks in childhood. Neuropsychologia, 42(8), 1029-1040. doi: 10.1016/j.neuropsychologia.2003.12.012

Rueda, M. R., Posner, M. I., \& Rothbart, M. K. (2005). The development of executive attention: Contributions to the emergence of self-regulation. Developmental Neuropsychology, 28(2), 573-594. doi:10. $1207 / \mathrm{s} 15326942 \mathrm{dn} 28022$

Shen, J., Reingold, E. M., \& Pomplun, M. (2000). Distractor ratio influences patterns of eye movements during visual search. Perception, 29, 241-250. doi:10.1068/p2933

Shen, J., Reingold, E. M., \& Pomplun, M. (2003). Guidance of eye movements during conjunctive visual search: The distractor-ratio effect. Canadian Journal of Experimental Psychology/Revue Canadienne De Psychologie Expérimentale, 57(2), 76-96. doi:10. 1037/h0087415

Shen, J., Elahipana, A., \& Reingold, E. M. (2007). Effects of context and instruction on the guidance of eye movements during a conjunctive visual search task. In R. G. van Gompel, M. H. Fischer, W. S. Murray, \& R. L. Hill (Eds.), Eye movements: A window on mind and brain (pp. 597-615). Amserdam: Elsevier. doi:10.1016/B978008044980-7/50030-6

Sobel, K. V., \& Cave, K. R. (2002). Roles of salience and strategy in conjunction search. Journal of Experimental Psychology: Human Perception And Performance, 28(5), 1055-1070. doi:10.1037/ 0096-1523.28.5.1055

Theeuwes, J. (1991). Exogenous and endogenous control of attention: The effect of visual onsets and offsets. Perception and Psychophysics, 49, 83-90.

Thompson, L. A., \& Massaro, D. W. (1989). Before you see it, you see its parts: Evidence for feature encoding and integration in preschool children and adults. Cognitive Psychology, 21, 334-362. doi:10. 1016/0010-0285(89)90012-1

Treisman, A., \& Gormican, S. (1988). Feature analysis in early vision: Evidence from search asymmetries. Psychological Review, 95, 1548.
Treisman, A., \& Souther, J. (1985). Search asymmetry: A diagnostic for preattentive processing of separable features. Journal of Experimental Psychology: General, 114, 285-310.

Treisman, A. M., \& Gelade, G. (1980). A feature-integration theory of attention. Cognitive Psychology, 12, 97-136.

Trick, L. M., \& Enns, J. T. (1998). Lifespan changes in attention: The visual search task. Cognitive Development, 13, 369-386.

Vales, C., \& Smith, L. B. (2015). Words, shape, visual search and visual working memory in 3-year-old children. Developmental Science, 18(1), 65-79. doi:10.1111/desc. 12179

Williams, D. E., \& Reingold, E. M. (2001). Preattentive guidance of eye movements during triple conjunction search tasks: The effects of feature discriminability and saccadic amplitude. Psychonomic Bulletin \& Review, 8(3), 476-488. doi:10.3758/BF03196182

Wolfe, J. M. (1994). Guided search 2.0: A revised model of visual search. Psychonomic Bulletin \& Review, 1, 202-238.

Wolfe, J. M. (2014). Approaches to visual search: feature integration theory and guided search. In K. Nobre \& S. Kastner (Eds.), The Oxford handbook of attention (pp. 11-50). Oxford: Oxford University Press.

Wolfe, J. M., Cave, K. R., \& Franzel, S. L. (1989). Guided search: An alternative to the feature integration model for visual search. Journal of Experimental Psychology: Human Perception and Performance, $15,419-433$.

Woods, A. J., Göksun, T., Chatterjee, A., Zelonis, S., Mehta, A., \& Smith, S. E. (2013). The development of organized visual search. Acta Psychologica, 143(2), 191-199. doi:10.1016/j.actpsy.2013.03.008

Yang, Y., \& Merrill, E. C. (2014). The impact of distracter-target similarity on contextual cueing effects of children and adults. Journal of Experimental Child Psychology, 121, 42-62. doi:10.1016/j.jecp. 2013.10.009

Yang, Y., \& Merrill, E. C. (2015). The impact of signal-to-noise ratio on contextual cueing in children and adults. Journal of Experimental Child Psychology, 132, 65-83. doi:10.1016/j.jecp.2014.12.005

Yantis, S., \& Jonides, J. (1984). Abrupt visual onsets and selective attention: Evidence from visual search. Journal of Experimental Psychology: Human Perception And Performance, 10(5), 601621. doi:10.1037/0096-1523.10.5.601

Zhuang, X., \& Papathomas, T. V. (2011). Cue relevance effects in conjunctive visual search: Cueing for location, color, and orientation. Journal of Vision, 11(7). doi:10.1167/11.7.6.

Zohary, E., \& Hochstein, S. (1989). How serial is serial processing in vision? Perception, 18, 191-200. 